\title{
Diagnóstico fenotípico de las inmunodeficiencias primarias en Antioquia, Colombia, 1994-2002
}

Carlos Julio Montoya, Julieta Henao, Helí Salgado, María M. Olivares, Juan A. López, Claudia Rugeles, José Luis Franco, Julio César Orrego, Diana M. García, Pablo J. Patiño

Grupo de Inmunodeficiencias Primarias, Corporación Biogénesis, Universidad de Antioquia, Medellín, Colombia.

La infección recurrente es una causa frecuente de consulta médica y puede deberse a un grupo heterogéneo de trastornos que incrementan la susceptibilidad a los microorganismos patógenos y oportunistas, como las deficiencias inmunológicas subyacentes. Con el fin de definir un tratamiento racional oportuno y orientar el diagnóstico molecular de las inmunodeficiencias primarias, se estableció un programa para el diagnóstico fenotípico de estas enfermedades en el departamento de Antioquia (Colombia), que comprende un análisis clínico y de laboratorio de los individuos que presentan infecciones recurrentes de evolución anormal. Entre el 1 de agosto de 1994 y el 31 de julio de 2002 se hizo el diagnóstico fenotípico de inmunodeficiencia primaria en 98 pacientes. Similar a lo informado en la literatura especializada, en esta región las deficiencias predominantes de anticuerpos fueron las más frecuentes $(40,8 \%)$ seguidas por las deficiencias combinadas $(21,4 \%)$. Esta caracterización fenotípica ha permitido iniciar rápidamente el tratamiento adecuado y, en algunos casos, comenzar los estudios funcionales y moleculares que conduzcan al diagnóstico molecular definitivo.

Palabras clave: inmunodeficiencias primarias, infección recurrente, Colombia.

Phenotypic diagnosis of primary immunodeficiency diseases in Antioquia, Colombia, 1994-2002

Recurrent infections are a frequent cause of medical visits. They can be due to a heterogeneous group of dysfunctions that increase the susceptibility to pathogenic and opportunistic microorganisms, such as immunological deficiencies. To define an opportune rational treatment and to guide the molecular diagnosis of primary immunodeficiency diseases, we establish a program for the phenotypic diagnosis of these illnesses in Antioquia, Colombia, including clinical and laboratory evaluations of patients who present recurrent infections with abnormal evolution. Between August 1, 1994 and July 31, 2002, phenotypic diagnosis of primary immunodeficiency was made in 98 patients. Similar to data reported in the literature, antibody deficiencies were the most frequent $(40.8 \%)$, followed by combined deficiencies $(21.4 \%)$. This phenotypic characterization has allowed for appropriate treatments for each patient and, in some cases, functional and molecular studies that can lead to a definite molecular diagnosis.

Key words: primary immunodeficiency diseases, recurrent infections, Colombia.

En condiciones normales, el contacto con los microorganismos patógenos durante el desarrollo de una infección hace que el sistema inmune

Correspondencia:

Carlos Julio Montoya, Carrera 51 D No. 62-29, oficina 206 , Universidad de Antioquia, Medellín, Colombia

Teléfonos: (572) 5106057 y 510 6078; Fax: (572) 5106047 cjmonto@catios.udea.edu.co

Recibido: 23/08/02; aceptado: 25/11/02 genere diversas respuestas efectivas contra ellos (1). Sin embargo, algunos trastornos estructurales o funcionales del hospedero impiden que algunos procesos infecciosos se controlen adecuadamente y se presenten con mayor frecuencia y severidad, dando origen a un cuadro clínico de infecciones recurrentes de comportamiento anormal en su evolución $(2,3)$. Aquellos individuos en los que se identifica este último cuadro clínico deberían 
someterse a un protocolo de análisis clínico y de laboratorio para tratar de confirmar una enfermedad de base que explique la recurrencia de las infecciones y permita definir el tratamiento pertinente (4).

Las inmunodeficiencias primarias (IDP) conforman uno de los grupos de enfermedades que se manifiestan característicamente por infecciones recurrentes de evolución anormal; se definen como experimentos de la naturaleza que afectan alguno de los genes que codifican para una proteína involucrada en el desarrollo y función del sistema inmune. Además de las infecciones recurrentes, otras manifestaciones menos frecuentes son las enfermedades alérgicas, autoinmunes y neoplásicas. Aunque aparentemente las IDP son poco frecuentes, muchas de ellas tienen una evolución muy severa que incluso puede ser letal, a menos que se realice un diagnóstico temprano y se instauren las medidas de tratamiento necesarias para cada caso $(5,6)$.

En las dos últimas décadas se ha presentado un avance muy acelerado en el desarrollo de las técnicas para la evaluación funcional y molecular del sistema inmune; esto ha permitido comprender más profundamente la estructura y fisiología de este sistema, así como establecer diagnósticos más precisos de las diferentes alteraciones inmunológicas y crear pautas de tratamiento más racionales. En el caso de las IDP este efecto ha sido muy notable, pues ha permitido establecer una clasificación fenotípica y molecular más precisa de estas enfermedades (7). Sin embargo, y debido a que existen cerca de 100 IDP bien definidas que afectan las diferentes ramas de la respuesta inmune, se requiere, en primer lugar, realizar una caracterización fenotípica de todos los casos sospechosos que permita determinar rápidamente un tratamiento que salve la vida del paciente y mejore su calidad de vida, pero que también oriente los estudios moleculares que se requieren para establecer un diagnóstico específico $(8,9)$.

Como una estrategia para evaluar la presencia de IDP en los pacientes con infecciones recurrentes anormales que consultan en los servicios de salud del departamento de Antioquia, el Grupo de
Inmunodeficiencias Primarias de la Universidad de Antioquia estableció a partir de agosto 1 de 1994 un programa piloto de vigilancia para la detección y estudio de los pacientes con infecciones recurrentes (10). Luego de descartar una causa no inmunológica de las infecciones en los casos remitidos (o una deficiencia secundaria como la infección por el virus de la inmunodeficiencia humana), los pacientes se someten a un protocolo de estudios clínicos y de laboratorio diseñado para establecer el diagnóstico fenotípico específico de una IDP y orientar el tratamiento correspondiente y la caracterización molecular definitiva (11)(figura 1).

El presente artículo resume los resultados más importantes obtenidos en ocho años de caracterización fenotípica de los pacientes con IDP, partiendo de los casos de infección recurrente anormal, y la forma como este programa ha contribuido al desarrollo de este campo científico en la región.

\section{Materiales y métodos}

\section{Programa para la detección y estudio de pacientes con infecciones recurrentes anormales}

Para dar respuesta a los frecuentes requerimientos de evaluación de pacientes con infecciones recurrentes provenientes de los servicios de salud de tercer nivel del departamento de Antioquia, el Grupo de Inmunodeficiencias Primarias de la Universidad de Antioquia diseñó en 1994 un programa de vigilancia para la detección y el estudio de los pacientes que presentaban infecciones recurrentes de una evolución característicamente anormal por su frecuencia, duración y severidad. La estructura de este programa y algunos resultados preliminares de su desarrollo han sido informados previamente (10-13).

Desde el inicio de las actividades de extensión el 1 de agosto de 1994, todos los pacientes remitidos fueron sometidos a una evaluación clínica inicial por parte de los especialistas en inmunología del grupo para establecer si realmente existían algunos de los criterios de anormalidad predeterminados (4) que permitieran considerar 


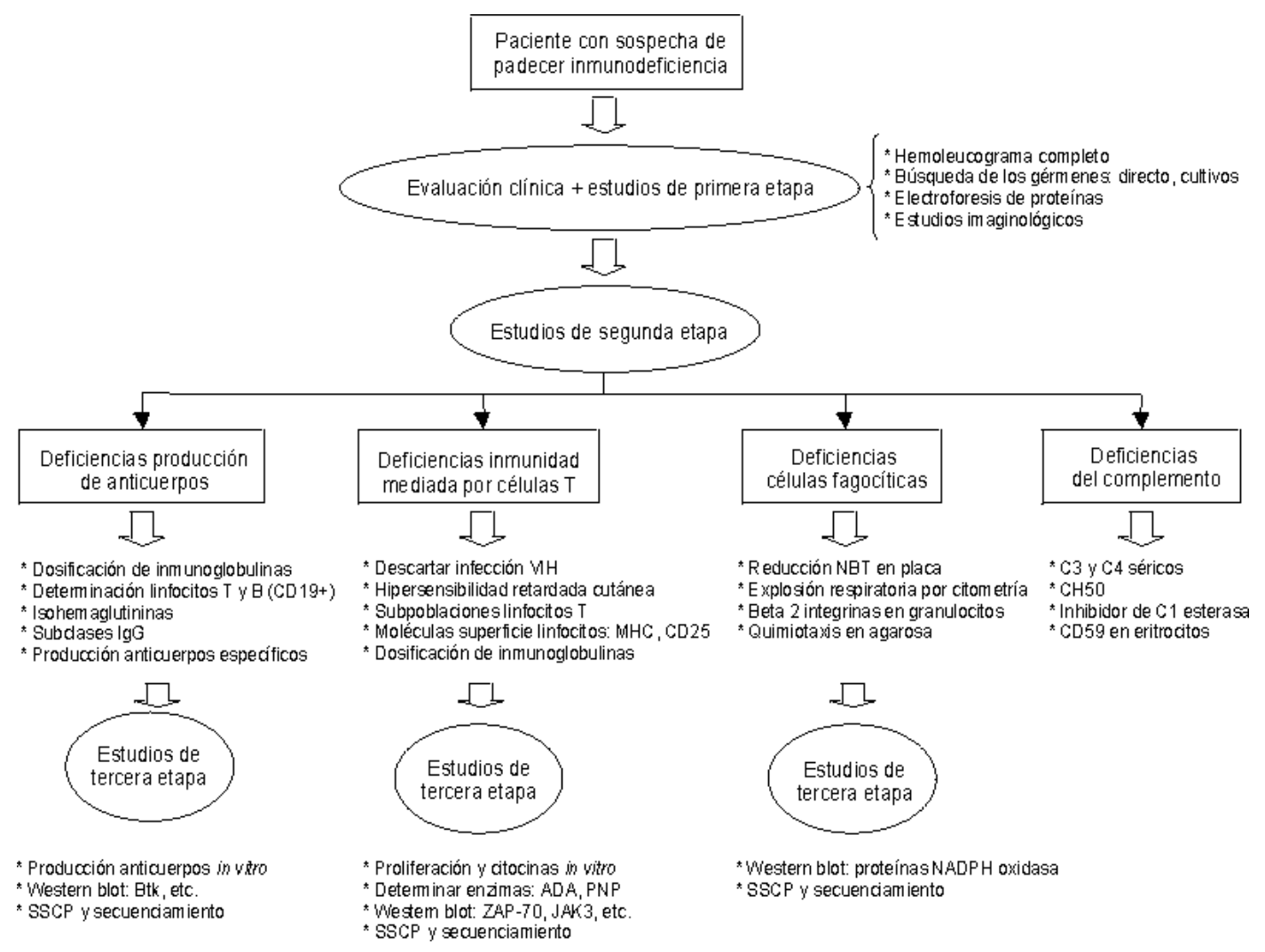

Figura 1. Protocolo para la evaluación de la inmunocompetencia (4). Diagrama de flujo recomendado para la evaluación de la inmunocompetencia en el paciente sospechoso de padecer inmunodeficiencia: estudios de laboratorio por etapas según niveles de complejidad y el tipo de defecto inmune sospechado.

IgG: inmunoglobulina G; VIH: virus de la inmunodeficiencia humana; MHC: complejo mayor de histocompatibilidad; Btk: tirosina cinasa de Bruton; SSCP: polimorfismos conformacionales en el ADN de cadena simple; ADA: adenosina deaminasa; PNP: purina nucleósido fosforilasa; NBT: nitroazul de tetrazolio; CH50: capacidad hemolítica 50 del suero.

el caso como un síndrome de infección recurrente anormal sospechoso de inmunodeficiencia; los más importantes de estos criterios son: tres o más episodios infecciosos, de moderados a graves, en el último año; antecedentes de hospitalización por infecciones severas en el último año; respuesta inadecuada al tratamiento antibiótico; aislamiento de un germen oportunista o inusual; aparición de secuelas o complicaciones; antecedentes de complicaciones por la aplicación de vacunas vivas; historia familiar de infección recurrente anormal, neoplasia hematológica, autoinmunidad o muerte en los primeros años de vida por una infección grave.
Además de descartar causas no inmunológicas de la recurrencia infecciosa (como cuerpos extraños, malformaciones congénitas, reflujo gastroesofágico, fibrosis quística), en esta primera consulta se definía el plan de estudios de laboratorio necesarios para evaluar cuantitativa y cualitativamente la inmunocompetencia en cada paciente en particular.

\section{Estudios de laboratorio para el diagnóstico de las IDP}

Cuando la información obtenida en la evaluación clínica inicial sugería la presencia de una IDP en el paciente, se realizaban los estudios de 
laboratorio por etapas consecutivas según los protocolos establecidos por el grupo (4). De acuerdo con los resultados de los estudios de la primera etapa, los gérmenes causales de las infecciones y la evolución de éstas, además de otras manifestaciones clínicas y rasgos asociados hallados en el examen físico (por ejemplo ataxia, telangiectasias, discromías cutáneas), los análisis de laboratorio se orientaron a la evaluación de la competencia de una de las cuatro ramas de la respuesta inmunológica: la producción de anticuerpos, la inmunidad mediada por las células $\mathrm{T}$, la función de las células fagocíticas y el sistema del complemento $(2,4)$. Los estudios de primera etapa comprenden pruebas como un hemoleucograma completo. Los estudios de segunda etapa son útiles para finalizar la caracterización fenotípica de la inmunodeficiencia y con los de tercera etapa se busca establecer el diagnóstico molecular definitivo cuando éste sea posible (figura 1).

\section{Clasificación de las IDP}

La definición de las IDP utilizada en este trabajo sigue las recomendaciones del grupo de expertos de la Organización Mundial de la Salud (8) y la clasificación fenotípica propuesta por el Grupo Latinoamericano de Inmunodeficiencias Primarias (LAGID) (14), que agrupa estos trastornos en seis clases principales: deficiencias combinadas (de la inmunidad celular y humoral); deficiencias predominantes de anticuerpos; deficiencias celulares y de anticuerpos asociadas con otros defectos mayores; deficiencias primarias de las células fagocíticas; síndromes de inmunodeficiencia asociados con disfunción de los fagocitos y las deficiencias congénitas del complemento.

Debido a que la mayoría de los pacientes diagnosticados todavía no tiene un diagnóstico molecular específico (porque éste aún no se conoce o porque no se han finalizado los estudios de tercera etapa), en esta publicación se utilizará la clasificación fenotípica. Los datos demográficos más importantes de cada paciente con inmunodeficiencia primaria se registraron en un formato definido por el LAGID para este fin (15) y en una base de datos computarizada que se desarrolló usando el programa Epi-Info versión 6.04.

\section{Tratamiento y seguimiento de los pacientes con IDP}

Con el objetivo de evitar recaídas y el desarrollo de secuelas, así como para mejorar la calidad de vida del paciente y brindar consejería genética cuando es posible-, después de realizar el diagnóstico de una IDP, todo paciente ingresa en forma voluntaria a un programa de atención y seguimiento periódico. El tratamiento farmacológico se determina según el diagnóstico fenotípico específico y siguiendo las recomendaciones de la literatura especializada $(16,17)$, así como los protocolos de tratamiento establecidos por el grupo (17-19). Los medicamentos prescritos son suministrados por la entidad prestadora de servicios de salud responsable de cada paciente. De acuerdo con el caso, se utilizan agentes antiinfecciosos (permanentes o profilácticos), gammaglobulina humana venosa, citocinas recombinantes (interferones I y II), entre otros $(16,20,21)$. Para algunas IDP letales se indica el trasplante de células madres hematopoyéticas, procedimiento que ya ha sido autorizado a nuestros primeros pacientes.

\section{Aspectos éticos}

Este programa no incluye ningún procedimiento que altere la estructura o el funcionamiento de los órganos de los pacientes evaluados y no se suministra ninguna sustancia no terapéutica para evaluar sus efectos en los individuos estudiados. El ingreso en el programa es voluntaria y los pacientes pueden retirarse en cualquier fase de su desarrollo. Las muestras que se utilizan para los estudios fenotípicos corresponden a sangre periférica obtenida por punción de una vena (idealmente del pliegue antecubital de alguno de los dos brazos). A todos los participantes o a sus tutores responsables se les explica en forma clara y sencilla los objetivos del programa y las implicaciones de los resultados que se obtendrán. Además, firman un consentimiento informado regido por la resolución 008430 de 1993 del Ministerio de Salud, que considera este tipo de investigación como de riesgo mínimo. Desde su inicio, el programa cuenta con la aprobación del Comité de Ética del Centro de Investigaciones Médicas de la Universidad de Antioquia. 


\section{Resultados}

Entre el 1 de agosto de 1994 y el 31 de julio de 2002 se evaluaron 698 pacientes remitidos, de los cuales, 411 (59\%) presentaban realmente criterios de anormalidad para considerarlos como portadores de un síndrome de infección recurrente anormal (SIRA). De acuerdo con las manifestaciones clínicas y los resultados del examen físico, se inició el programa de estudios por etapas de complejidad para confirmar la presencia de una deficiencia inmunológica y establecer su clasificación fenotípica. En 98 individuos se confirmó la presencia de una inmunodeficiencia primaria; 48 pacientes padecían de una inmunodeficiencia secundaria y el resto de casos de SIRA tenían una causa no inmunológica que predisponía a las infecciones recurrentes (datos no mostrados).

\section{Diagnósticos fenotípicos de IDP}

De acuerdo con la clasificación fenotípica propuesta por el LAGID (14), de los 98 casos de IDP, la mayoría correspondía a una deficiencia predominante de anticuerpos (40,8\%); le seguían en frecuencia las deficiencias combinadas $(21,5 \%)$, los síndromes de inmunodeficiencia asociados con disfunción de los fagocitos (15,3\%) y las deficiencias celulares y de anticuerpos

Cuadro 1. Clasificación por fenotipos de las 98 IDP identificadas en los pacientes del Grupo de Inmunodeficiencias Primarias de la Universidad de Antioquia.

\begin{tabular}{|c|c|c|c|c|}
\hline Fenotipo predominante & Hombres & Mujeres & Total & \\
\hline \multicolumn{5}{|l|}{ Deficiencias predominantes de anticuerpos } \\
\hline Hipogammaglobulinemia transitoria de la infancia & 10 & 4 & 14 & \\
\hline Inmunodeficiencia común variable & 2 & 9 & 11 & \\
\hline Agammaglobulinemia congénita & 8 & 0 & 8 & \\
\hline Déficit de $\lg A$ & 2 & 1 & 3 & \\
\hline Síndrome de hiper-IgM & 1 & 1 & 2 & \\
\hline Deficiencias de anticuerpos con Ig normales & 0 & 0 & 1 & \\
\hline Inmunodeficiencia común variable con timoma & 1 & 0 & 1 & \\
\hline Subtotal (\%) & & & & $40(40,8)$ \\
\hline \multicolumn{5}{|l|}{ Deficiencias combinadas } \\
\hline Inmunodeficiencia combinada severa & 5 & 8 & 13 & \\
\hline Inmunodeficiencia combinada no severa & 2 & 0 & 2 & \\
\hline Inmunodeficiencia celular con Igs normales & 1 & 5 & 6 & \\
\hline Subtotal (\%) & & & & $21(21,5)$ \\
\hline \multicolumn{5}{|l|}{$\begin{array}{l}\text { Deficiencias celulares y de anticuerpos asociadas } \\
\text { con otros defectos mayores }\end{array}$} \\
\hline Candidiasis mucocutánea crónica & 4 & 2 & 6 & \\
\hline Síndrome de Wiskott Aldrich & 4 & 0 & 4 & \\
\hline Ataxia telangiectasia & 1 & 1 & 2 & \\
\hline Anomalía de DiGeorge & 0 & 2 & 2 & \\
\hline Enanismo con extremidades cortas & 1 & 0 & 1 & \\
\hline Subtotal| & & & & $15(15,3)$ \\
\hline \multicolumn{5}{|l|}{$\begin{array}{l}\text { Síndromes de inmunodeficiencia asociados con } \\
\text { disfunción de los fagocitos }\end{array}$} \\
\hline Síndrome de hiper-IgE con infecciones recurrentes & 6 & 4 & 10 & \\
\hline Síndrome de Chediak Higashi & 2 & 3 & 5 & \\
\hline Subtotal & & & & $15(15,3)$ \\
\hline \multicolumn{5}{|l|}{ Defectos primarios de las células fagocíticas } \\
\hline Enfermedad granulomatosa crónica & 4 & 0 & 4 & \\
\hline Neutropenia congénita severa & 1 & 1 & 2 & \\
\hline Subtotal & & & & $6(6,1)$ \\
\hline \multicolumn{5}{|l|}{ Deficiencias del complemento } \\
\hline Edema angioneurótico hereditario & 0 & 1 & 1 & \\
\hline Subtotal & & & & $1(1,0)$ \\
\hline Total & & 55 & 43 & $98100 \%$ \\
\hline
\end{tabular}


asociadas a defectos mayores (15,3\%). La más baja frecuencia se observó en los defectos primarios de las células fagocíticas $(6,1 \%)$ y las deficiencias congénitas del complemento $(1,0 \%)$ (cuadro 1).

Con respecto al fenotipo específico diagnosticado en cada paciente, se observó que las IDP más comunes eran la hipogammaglobulinemia transitoria de la infancia (14/98), la inmunodeficiencia combinada severa (13/98), la inmunodeficiencia común variable (11/98), el síndrome de hiper-lgE con infecciones recurrentes (10/98) y la agammaglobulinemia congénita (8/98). Sólo tres pacientes fueron caracterizados con el fenotipo de deficiencia de lgA, una de las IDP más frecuentes en la población general.

Se debe resaltar que, en general, existieron 20 diagnósticos fenotípicos diferentes con casos correspondientes a cada uno de los seis grupos del LAGID, reflejo de la gran heterogeneidad fenotípica de las IDP. Sin embargo, esto también indica que en nuestro medio existe una buena capacidad diagnóstica para la caracterización de las diferentes IDP.

En los pacientes con IDP se observó un ligero predominio de los pacientes de sexo masculino (56\%; 55/98) sobre los de sexo femenino (44\%; 43/98). Se pudo establecer el antecedente familiar de una misma IDP en nueve casos, la mayoría en hermanos. Este antecedente fue más frecuente en la inmunodeficiencia combinada severa ( 3 casos), pero también se encontró en la enfermedad granulomatosa crónica, en la agammaglobulinemia congénita, en la ataxia telangiectasia y en la inmunodeficiencia común variable.

De los casos confirmados de IDP, 27 han fallecido por complicaciones infecciosas severas relacionadas con su enfermedad de base (índice de letalidad del $27,6 \% ; 27 / 98)$. Las IDP de mayor letalidad fueron la inmunodeficiencia combinada severa (11 de 13 pacientes) y la agammaglobulinemia congénita (3 muertes).

\section{Seguimiento y tratamiento de las IDP}

De los pacientes con IDP diagnosticada que se sabe están con vida en el momento de escribir este informe, 55 asisten periódicamente a las citas de control ambulatorio con los especialistas del Grupo de Inmunodeficiencias Primarias; en estas citas se analiza la evolución clínica de cada paciente, la respuesta a la terapia y se planifican otros estudios de diagnóstico y control; además, se da educación y asesoría al paciente y a los familiares responsables de ellos. El medicamento utilizado con más frecuencia para el tratamiento de nuestros pacientes con IDP es la gammaglobulina humana endovenosa; 29 pacientes con fenotipos muy heterogéneos de IDP han recibido sin interrupción estos preparados de gammaglobulina a una dosis de $400 \mathrm{mg} / \mathrm{kg} / \mathrm{mes}$. La mayoría de los pacientes tratados con gammaglobulina presentan deficiencias cuantitativas graves en la producción de anticuerpos (diez con inmunodeficiencia común variable, siete con agammaglobulinemia congénita y cuatro con deficiencia combinada severa), pero también se ha administrado a pacientes con otras IDP con alteraciones cualitativas en la secreción de anticuerpos como los síndromes de WiskottAldrich, hiper-lgE e hiper-lgM.

Hasta 2001, no existía en la región la posibilidad de realizar una terapia de reemplazo con trasplante de células madres hematopoyéticas para las deficiencias de carácter letal, situación que parcialmente explica la alta mortalidad de algunas IDP como la inmunodeficiencia combinada severa. Entre agosto de 2001 y el momento de realizar este informe se han realizado en Medellín (Servicio de Hematología del Hospital Universitario San Vicente de Paúl) los dos primeros trasplantes de células madres, provenientes de sangre de cordón umbilical, en dos pacientes con inmunodeficiencia severa combinada diagnosticados en este programa.

\section{Discusión}

Las IDP son enfermedades muy complejas tanto en su origen como en las manifestaciones clínicas que las caracterizan; su principal síntoma es un cuadro de infecciones recurrentes de etiología y severidad variables que dependen de la causa molecular subyacente. El desconocimiento de la existencia de estas enfermedades por el personal de atención en salud hace que, en su evolución, la morbilidad sea elevada, con secuelas graves, y que la muerte precoz sea la regla. Por eso, el 
desarrollo de centros regionales para el diagnóstico y tratamiento de estos trastornos podría tener un impacto importante en la salud de los pacientes con alteraciones en la función del sistema inmune. Hasta el momento, el Grupo de Inmunodeficiencias Primarias de la Universidad de Antioquia es el único centro en Colombia con un programa organizado para la detección, el diagnóstico fenotípico y el tratamiento de las IDP; también es uno de los pocos centros en Latinoamérica con esta capacidad. Este es el estudio más completo que se ha publicado sobre el diagnóstico fenotípico de las IDP en Colombia; los datos de nuestro trabajo sirvieron unos años atrás como fuente para la caracterización del problema en Latinoamérica (22).

En el área de las IDP, no han sido muchos los intentos de crear sistemas de remisión, diagnóstico y atención para los pacientes. Se han informado las experiencias de países como Japón (23), Suecia (24), Inglaterra (25), España $(26,27)$, Suiza $(28,29)$, Italia (30), Australia (31), Sudáfrica (32) y Brasil (33). También ha sido creciente el interés de algunas agremiaciones de carácter internacional por difundir la importancia de las IDP y estimular la creación de centros nacionales y regionales de referencia para el estudio y tratamiento de los pacientes: PAGID (Grupo Panamericano), LAGID (Grupo Latinoamericano), ESID (Grupo Europeo).

En los últimos 15 años, el área de las IDP ha sufrido los efectos del creciente conocimiento de la inmunología básica, por lo que se ha observado un aumento destacado en la caracterización de los defectos moleculares responsables de un buen número de IDP (7), han aparecido nuevas propuestas de protocolos de diagnóstico y tratamiento internacionales $(8,21,34)$ y se han iniciado los primeros ensayos de terapia génica para las inmunodeficiencias severas (35). Todo lo anterior resalta la importancia que han adquirido los defectos primarios de la respuesta inmune en el campo de la atención médica, así como la relevancia de un programa dirigido a detectar a los individuos con estas enfermedades mediante la vigilancia de una de sus manifesta-ciones clínicas más comunes, la infección recurrente.
En cuanto a la distribución fenotípica de las IDP caracterizadas por nuestro grupo, las deficiencias predominantes de anticuerpos fueron las más frecuentes $(40,8 \%)$, lo que concuerda con lo relatado en la literatura especializada (5). En conjunto, las alteraciones en la función de las células fagocíticas se encontraron en un porcentaje $(21,4 \%)$ similar al establecido en otros informes (18\%) (5). Entre las inmunodeficiencias caracterizadas merece una mención especial el síndrome de hiper-lgE con infecciones recurrentes, de la cual hemos identificado diez casos; esta rara enfermedad ha sido recientemente redefinida como una alteración multisistémica, de la cual sólo alrededor de 200 casos han sido informados en el mundo (36). Las inmunodeficiencias celulares (combinadas y puras) y las deficiencias del complemento también se encontraron en un porcentaje acorde con lo observado en otros informes.

Sólo se han diagnosticado tres pacientes con fenotipo de deficiencia de $\lg A$; si bien ésta ha sido la inmunodeficiencia primaria más frecuentemente informada, con una prevalencia entre 1:300 y 1:700 individuos (37), su caracterización se ha realizado fundamentalmente en comunidades normales y bancos de sangre, pues la mayoría de los afectados son asintomáticos. En nuestro caso, los tres pacientes diagnosticados eran sintomáticos, pues nuestro programa sólo evalúa sujetos con manifestaciones clínicas de moderadas a severas.

Otro aporte importante de este programa es la detección de casos familiares de IDP, que permite suministrar en algunas oportunidades consejería genética para evitar que se presenten nuevos individuos afectados en un mismo grupo familiar. Algunas IDP letales, como la enfermedad granulomatosa crónica y la inmunodeficiencia severa combinada, si no se diagnostican oportunamente pueden presentar una alta letalidad en una misma familia (38).

Contar con un servicio de estas características ha permitido hacer el diagnóstico temprano en muchos casos de IDP, antes de que los pacientes desarrollen secuelas graves que afecten su calidad de vida. Para la gravedad de la mayoría 
de las IDP, consideramos que la mortalidad ha sido baja, en directa relación con la terapia preventiva o de sustitución y la evaluación permanente de los pacientes. En particular, queremos resaltar la terapia con gammaglobulina humana venosa, un producto con más de 20 años de utilidad demostrada en las IDP y otras alteraciones inmunológicas, pero poco utilizado en nuestro medio debido a su alto costo y al desconocimiento de sus indicaciones y forma de aplicación para evitar las reacciones secundarias. Al respecto, hemos elaborado para el LAGID un documento sobre las indicaciones y uso correcto de la gammaglobulina venosa (17). También se debe resaltar la promoción de un grupo especializado en la terapia con células madres hematopoyéticas de trastornos tan severos como las IDP.

En conclusión, el desarrollo de este programa de atención clínica y diagnóstico fenotípico y molecular ha permitido la caracterización de un número muy importante de pacientes con IDP, trastornos considerados poco frecuentes en la comunidad general. Contar con un grupo tan variado de diagnósticos fenotípicos ha permitido la creación de un amplio campo de investigación básica y clínica que potencialmente puede tener profundas repercusiones en el fortalecimiento del área de la inmunología en el país.

\section{Agradecimientos}

Este trabajo fue realizado gracias al apoyo de la Dirección Seccional de Salud de Antioquia, del Comité para el Desarrollo de la Investigación (CODI) de la Universidad de Antioquia y de Colciencias.

\section{Referencias}

1. Abbas AK, Lichtman AH, Pober JS. Immunity to microbes. En: Abbas AK, Lichtman AH, Pober JS, editores. Cellular and molecular immunology. Philadelphia: W.B. Saunders Company; 2000. p.343-62.

2. Paul ME, Shearer WT. Approach to the evaluation of the immunodeficient patient. En: Rich RR, Fleisher TA, Schwartz BD, editores. Clinical immunology. Principles and practice. St. Louis: Mosby; 1996. p.609-20.

3. García D, Patiño PJ, Salgado H, López JA, Montoya CJ, Pérez JE. Evaluación del paciente con inmunodeficiencia. Síndrome de infección recurrente patológica. Medicina y Laboratorio 1997;7:545-75.
4. Montoya CJ, Salgado H, Olivares MM, Patiño PJ. Evaluación de la respuesta inmune en el paciente con infección recurrente sospechoso de padecer inmunodeficiencia. Salud UIS 2000;32:105-11.

5. Conley ME, Stiehm ER. Immunodeficiency disorders: general considerations. En: Stiehm ER, editor. Immunologyc disorders in infants and children. Philadelphia: W.B. Saunders Company; 1996. p.201-52.

6. Buckley RH. Primary immunodeficiency diseases. En: Paul WE, editor. Fundamental immunology. Philadelphia: Lippincott-Raven Publishers; 1999. p.1427-53.

7. Smith CIE, Ochs HD, Puck JM. Genetically determined immunodeficiency diseases: a perspective. En: Ochs HD, Smith CIE, Puck JM, editores. Primary immunodeficiency diseases. A molecular and genetic approach. Oxford: Oxford University Press; 1999. p.3-11.

8. WHO Scientific Group. Primary immunodeficiency diseases. Report of a WHO scientific group. Clin Exp Immunol 1995;99(Suppl1):1-24.

9. Holland MS, Gallin JI. Evaluation of the patient with suspected immunodeficiency. En: Mandell, Douglas, Bennett, editores. Principles and practice of infectious diseases. New York: Churchill Livingstone; 1995. p.14958.

10. García D, Montoya CJ, Salgado H, Gómez RD, Botero JH, Patiño PJ, et al. Detección y manejo de infección recurrente patológica. Estudio piloto, Antioquia 1994-1995. Boletín Epidemiológico de Antioquia 1995;20:167-71.

11. García D, Montoya CJ, Salgado H, Patiño PJ, López JA, Orrego JC, et al. Caracterización de pacientes con inmunodeficiencias primarias por medio de un programa de vigilancia epidemiológica para la infección recurrente. Estudio Piloto, Medellín, 1994-1999. Acta Méd Colomb 2001;26:50-9.

12. García D, Montoya CJ, Salgado H, Gómez RD, Patiño PJ, Botero JH, et al. Sistema de vigilancia epidemiológica para el síndrome de infección recurrente patológica (SIRP). Boletín Epidemiológico de Antioquia 1995;20:157-66.

13. Montoya CJ. Actualización del diagnóstico de inmunodeficiencias primarias en Antioquia: programa para la detección y manejo del síndrome de infección recurrente. Rev Asoc Colomb Alerg Asma Inmunol 1999;8:29-33.

14. Latin American Group of Primary Immunodeficiencies, LAGID. Clasificación fenotípica de las inmunodeficiencias primarias. Boletín LAGID 1997;1:11-3.

15. Latin American Group of Primary Immuno-deficiencies, LAGID. Registro de inmunodeficiencias primarias. Boletín LAGID 1997;1:9-10.

16. Stiehm ER. Conventional therapy of primary immunodeficiency diseases. En: Ochs HD, Smith CIE, Puck JM, editores. Primary immunodeficiency diseases. 
A molecular approach. Oxford: Oxford University Press; 1999. p.448-58.

17. Montoya CJ, Sorensen RU. Lecciones sobre el uso de gammaglobulina humana endovenosa. Boletín LAGID, 23 de febrero de 2001. Disponible [on-line]: http:// www.lagid.Isuhsc.edu/Tratamientos/971-010.htm 2001.

18. Montoya CJ, Salgado H, Henao J, Orrego JC, Patiño PJ. Guía de estudio y manejo del paciente sospechoso de alteraciones en la respuesta inmune humoral específica. Rev Asoc Colomb Alerg Asma Inmunol 1999; 8:32-5.

19. Salgado H, Montoya CJ, Henao J, Orrego JC, López JA, Patiño PJ. Guía de estudio y manejo del paciente sospechoso de presentar alteraciones en la respuesta inmune celular específica. Rev Asoc Colomb Alerg Asma Inmunol 2000;9:9-13.

20. Polmar SH, Sorensen RU. Immunoglobulin replacement therapy in primary immunodeficiency diseases. En: Rich RR, Fleisher TA, Schwartz BD, editores. Clinical immunolgy: principles and practice. St. Louis: Mosby; 1996. p.1865-73.

21. IUIS Scientific Committee. Primary immunodeficiency diseases. Clin Exp Immunol 1999;118:1-28.

22. Zelazko M, Carneiro-Sampaio $\mathbf{M}$, Cornejo $\mathbf{M}$, García D, Porras O, Berrón R, et al. Primary immunodeficiency diseases in Latin America: first report from eight countries participating in the LAGID. J Clin Immunol 1998;18:161-6.

23. Hayakawa H, Iwata T, Yata J, Kobayashi N. Primary immunodeficiency syndrome in Japan I: overview of a nationwide survey of primary immunodeficiency syndrome. J Clin Immunol 1981;1:31-9.

24. Fasth A. Primary immunodeficiency disorders in Sweden: cases among children 1974-1979. J Clin Immunol 1982;2:86-92.

25. Roberton DM, Shelton MJ, Hosking CS. Incidence of Primary immunodeficiency disorders in childhood (abstract). Fifth International Congress of Immunology, 1983.

26. Español T, García R, García SJA. Inmunodeficiencias primarias en Cataluña. Inmunología 1985;4:50-2.

27. Matamoros N, Mila J, Español T, Raga S, Fontan G. Primary immunodeficiency syndrome in Spain: first report of the national registry in children and adults. $J$ Clin Immunol 1997;17:333-9.

28.Ryser O, Morell A, Hitzing WH. Primary immunodeficiencies in Switzerland: first report of the national registry in adults and children. J Clin Immunol 1988;8:47985.

29. Affentranger $\mathbf{P}$, Morell A, Spath $\mathbf{P}$, Seger R. Registry of primary immunodeficiencies in Switzerland. Immunodeficiency 1993;4:193-5.

30. Luzi G, Pesce AM, Rinaldi S. Primary immunodeficiencies in Italy. Data revised from the Italian register of immunodeficiencies, IRID (1977-88). Immunol Clin 1989;8:45-54.

31. Baumgart KW, Britton WJ, Kemp A, French $\mathbf{M}$, Roberton D. The spectrum of primary immunodeficiency disorders in Australia. J Allergy Clin Immunol 1997;100: 415-23.

32. Eley B, Beatty D. Primary immunodeficiency diseases in Cape Town. ACl International 2000;12:267-70.

33. Grumach AS, Duarte AJS, Bellinati-Pires R, Pastorino AC, Jacob CMA, Diogo CL, et al. Brazilian report on primary immunodeficiencies in children: 166 cases studied over a follow-up time of 15 years. J Clin Immunol 1997;17:340-5.

34. Conley ME, Notarangelo LD, Etzioni A. Diagnostic criteria for primary immunodeficiencies. Clin Immunol 1999;93:190-7.

35. Candotti F. The potential for therapy of immune disorders with gene theraphy. Pediatr Clin North Am 2000;47:1389-407.

36. Grimbacher B, Holland SM, Gallin JI, Greenberg F, Hill SC, Malech HL, et al. Hyper-IgE syndrome with recurrent infections. An autosomal dominant multisystem disorder. N Engl J Med 1999;340:692-702.

37. Cunningham-Rundles $\mathbf{C}$. Disorders of the $\lg A$ system. En: Stiehm ER, editor. Immunologic disorders in infants and children. Philadelphia: W.B. Saunders Company; 1996. p.423-42.

38. Montoya CJ, Ayala A. Enfermedad granulomatosa crónica ligada al cromosoma $X: 4$ casos familiares fatales. Rev Asoc Colomb Alerg Asma Inmunol 1999;8: 59-61. 